\title{
APPLICATION OF ARTIFICIAL INTELLIGENCE TECHNIQUES IN UNINHABITED AERIAL VEHICLE FLIGHT
}

\author{
Warren R. Dufrene, Jr., Graduate School of Computer and Information Sciences, Nova Southeastern \\ University, Ft. Lauderdale, FL
}

\begin{abstract}
This paper describes the development of an application of Artificial Intelligence (AD) for Unmanned Aerial Vehicle (UAV) control. The project was done as part of the requirements for a class in AI at NOVA Southeastern University and a beginning project at NASA Wallops Flight Facility for a resilient, robust, and intelligent UAV flight control system. A method is outlined which allows a base level application for applying an Artificial Intelligence method, Fuzzy Logic, to aspects of Control Logic for UAV flight. One element of UAV flight, automated altitude hold, has been implemented and preliminary results displayed.
\end{abstract}

\section{Introduction}

The attempt of man to fly UAV's has been going on for a long time. Use of UAV's can be traced back to use in World War I. Current research continues at institutions such as Berkeley, MIT, Georgia Tech, and many government organizations such as NASA, NOAA, the Navy, Air Force, and the Army. Universities in countries all over the world continue to research unmanned aerial flight and continue to push the envelope of the applications for Artificial Intelligence and conventional control methods, which guide the aircraft.

Current research efforts are looking at advancing the UAV to full autonomous operation and flight. While there seem to be many definitions of what the autonomy encompasses, each discipline has highlighted its meaning and benefits.

Liu discusses a good outline of the problems involved in achieving control of the complex systems today [1]. He outlines the heterogeneous modeling and design of an advanced control system approach. One of the key problems faced is the fact that the systems and sensors involved are heterogeneous in nature. These systems have to be integrated and resolved into a working system. For the most part, this will be true for most complex UAV systems, which attempt full autonomy.

\section{Background}

New applications of AI, which include Intelligent Agents, are providing new areas of research [2]. Research has expanded during the past five years within the applications of Artificial Intelligence (AI) methods applied to the concepts of flight for the Unmanned Aerial Vehicle (UAV). The UAV can be described as any platform that is operated without on board humañ occupation. Systems, which exist today, include helicopters, airplanes, balloons, blimps, and even satellites. Their autonomy varies from human interaction from a remote console to fully autonomous takeoff and landing. Earlier research, conducted by Handelman and Stengel, applied AI methods such as expert systems and schedulers, which applied rule-based systems [3]. These systems attempted to formulate the art of flying an aircraft into logical series of events to maintain control of specific functions of the aircraft. This concept will still be a key component with systems designed for future use.

The initial concept of using an Expert System to control an aircraft seems simple at first but proves difficult to apply. Some of the problems that still exist today include the fact that an expert pilot's decision-making process is difficult to imitate with computers. The dynamic environment and dynamic conditions affecting the aircraft are areas that have to be adapted to such an Expert System.

Later research efforts began to divide the many tasks involved in the control of UAV flight into manageable steps. Concentrated efforts of applying logic schemes similar to Fuzzy Logic and Neural Networks, are being applied to improve the mathematical solutions for flight control. Dr. 
Anthony J. Calise, professor of aerospace engineering at Georgia Tech stated that, "several areas of control for UAV's can be adapted to the use of Neural Networks" [4]. The author gives several accounts of the advantages of applying Neural Networks in addition to the normal control methods of a UAV through an integration of these systems.

Current research efforts are starting to evolve the application of AI approaches into Hybrid Systems. Researchers are thinking about the expected advances in aerial vehicle control. Dr. Norman Fitz-Coy, professor of mechanical engineering at the University of Florida, stated: "Ultimately, it is expected that an ideal flight control system for an adaptive wing Miniature Aerial Vehicle (MAV) will use soft computing techniques, such as fuzzy logic, neural networks, genetic algorithms, pattern recognitions, or knowledge-based systems" [5].

Past research efforts are being modified to include an integration of more AI methods. These current research efforts are the interesting advancements taking place. How does a machine fly an aircraft like a human can? What does a human think about in the process of reacting to the environment? Can a machine really "think" enough to fly a UAV fully autonomously? These are questions pushing UAV research.

\section{The Problem Outlined}

Research of the applications of AI is coming to a point where the approaches, algorithms and models need to be applied for further understanding of their usefulness and applicability towards any given project for a UAV [6], [7]. Most of the research papers reviewed gave excellent theory but did not give enough information on the mechanics or steps for applying the methods outlined to begin a research effort.

The focus of this research has been to outline some of the AI techniques used for UAV flight control and discuss some of the tools used to apply AI techniques. The intent is to succeed with the implementation of applying AI techniques towards actually controlling different aspects of UAV flight. These tools and techniques serve as the project's basic start.

\section{The Approach}

There is an increase in research and papers on accomplishments and proposed research under the subject of unmanned flight. The field was narrowed down to include $\mathrm{AI}$ methods that could be applied easily in a lab environment.

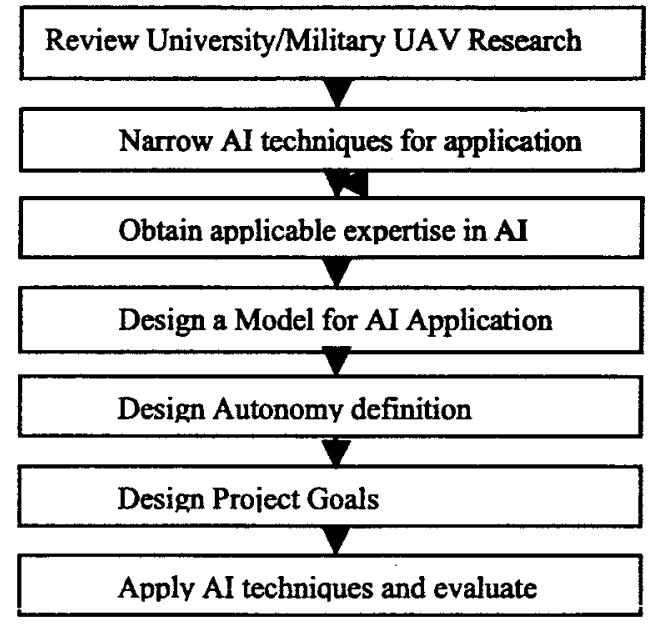

Figure 1. Design Approach

During the completion of the steps in Figure 1, the following work was accomplished. The decision to use JAVA was due to a requirement for the AI class. Although the use of a new language was slow at first, this programming language environment proved to be very beneficial for this type of project. The following summary is given for the general approach:

a. The first approach to the problem was to research the past and current efforts of UAV flight control at universities. This research was done on the web through sites and journal articles.

b. Research was done after isolating the choice for elevator control to maintain altitude. An attempt was made to find a fuzzy logic program or applet written in the JAVA language.

c. While doing the research several books were purchased for learning JAVA. The Sun JAVA site was also used, as recommended, for the initial understanding of the format and structure to the JAVA language.

d. The approach for the project was to learn basic JAVA programming techniques for reading 
simulated UAV flight files, converting these files if needed, and running or modifying a Fuzzy Logic application.

e. Three strong candidates for usable Fuzzy Logic JAVA programs were found:

1). The first package was a complete JAVA class package developed for use within a JAVA program. This program is called Fuzzy Engine by Professor Edward Sazonov and can be found a t $\quad t$ h e a d d res s : http://www.csee.wvu.edu/ esazonov/FuzzyEngi ne.htm. This package was reviewed and set aside as a future application for more advanced work.

2). The primary candidate was a program called FIT written for Fuzzy control by Gerald A. De Jong (gerald@beautifulcode.nl.) retrieved from: (http://www.beautifulcode.nl).

This package included a program already designed for a fan controller. The code was a good candidate for modification to a simple elevator controller for the UAV.

$3)$. The second candidate was a commercial package with free demo download called FuzzyTech 5 which can be retrieved from: (http://www.fuzzytech.com/index.htm). The FuzzyTech package allowed input of a data file into a Fuzzy Controller that can be designed within the program's own Graphical User Interface. Work was done with this package also and some of it will be presented. The licensed package will also convert the design to code in JAVA or C.

The description of the contents of Figure 1, are discussed in greater detail.

\section{Review University UAV Research}

The key concepts reviewed included work done by Berkeley, University of Florida, and several other universities. The research papers provided key ideas for the application of the actual concepts to use Fuzzy Logic as a base AI approach. While success was not guaranteed, an educated evaluation was done to review the applicability.
The main attempt was to duplicate linear control methods by using a fuzzy controller.

\section{Narrow AI techniques for application}

The approaches seen in most of the research included Expert Systems, Neural Networks, Fuzzy Logic, and Hybrid combinations of these and PID control schemes. The AI approaches selected for use were Fuzzy Logic, Expert System, and later a combination of Neural / Fuzzy for adaptive capability.

\section{Obtain applicable expertise in AI Methods}

The first AI approach employed during the project was Fuzzy Logic. This technique was used to control the altitude hold of a simulated UAV. Two applications were performed. The first application involved writing JAVA code to simulate a flight using calibrated steps of altitude climbs. The second application involved learning a demo copy of a commercial program for designing a Fuzzy Control System (FuzzyTech 5.x). The output data from the first application was used for input into the demo program. The output data file from FuzzyTech was imported into an Excel spreadsheet for comparison against the input data.

\section{Design a Model for AI Application}

A model was needed to allow a confined implementation of a simple Fuzzy control scheme. The chosen altitude hold was used with an added input for acceleration control. The acceleration control was for future work involving degrees of power needed during different scenarios of flight (take-off, level flight, disturbed flight, and approach).

The design for the fuzzy controller is outlined in Figure 2. The first system only included altitude as an input and elevator as the output. It proved to be a simple and stable control scheme.

Acceleration was added to give future degrees of control for a throttle output to compensate for levels of dynamic change in conditions of the flight envelope. 


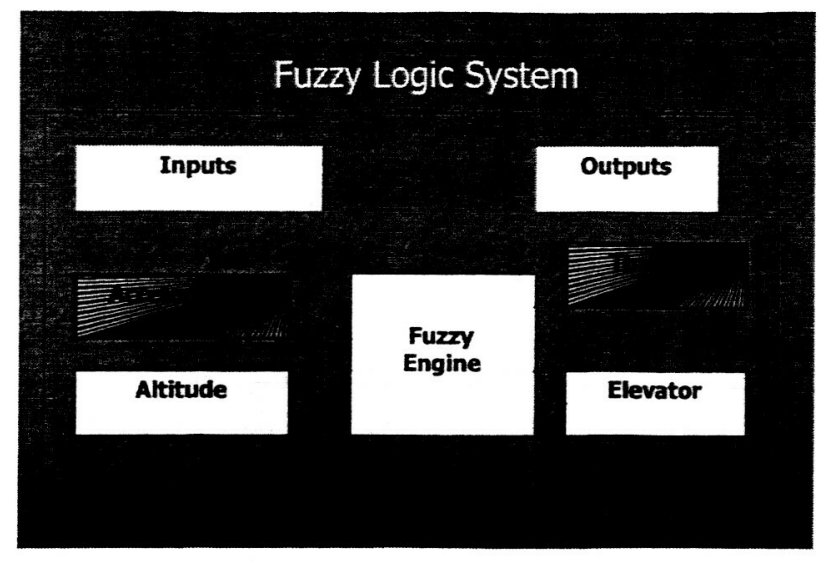

Figure 2. Fuzzy Controller

\section{Design Autonomy definition}

The definition for autonomy for this project has been defined as the creation of a craft (which includes the smallest breakdown of agents) which can perform its pre-determined function without any additional human interaction. This definition will be carried out by the loading of a mission plan for the craft to carry out.

Figure 3 displays the objective of the autonomy. This will apply to all elements of the design. The simple altitude hold will follow the same criteria. The mission plan will be the desired altitude to hold. The mission objective will become the desired altitude. The fuzzy controller will control the UAV Craft and a review is still done inherently by the change in input to the fuzzy controller.

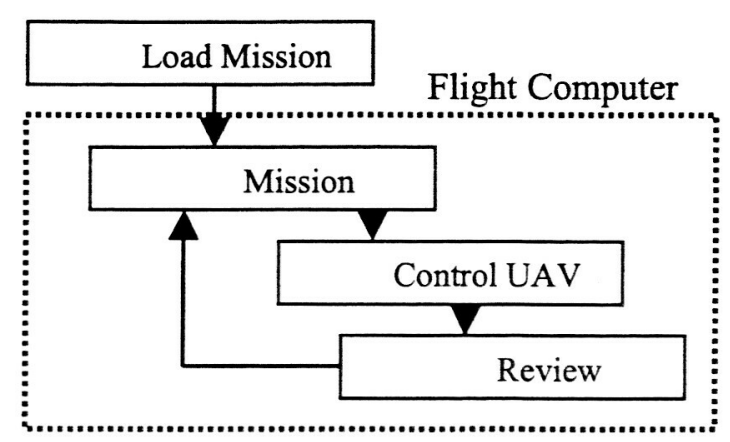

Figure 3. Autonomy Objective

\section{Design Project Goals}

The project goals flow diagram in Figure 4 outlines the required steps attempted to complete the project. The choice was made to work with existing JAVA code and implement an off-the-shelf application. This was decided so the control output effects could be compared against each other for verification of correct response behavior.

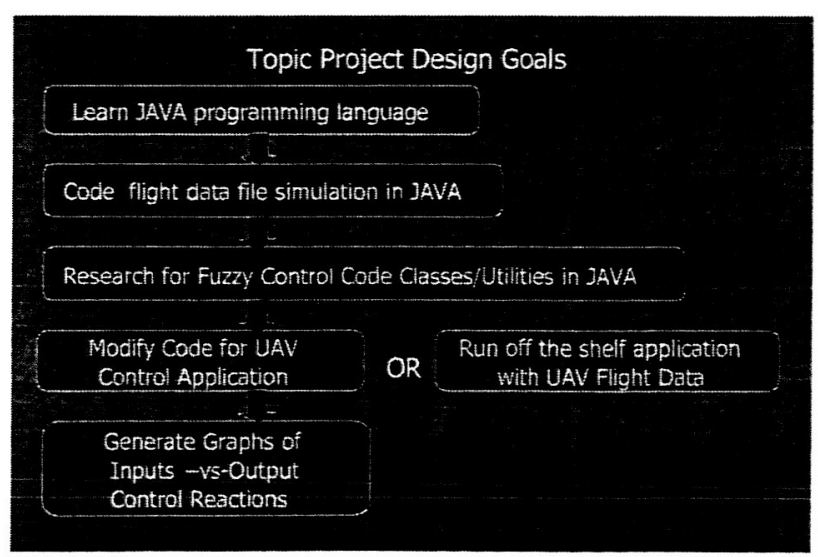

Figure 4. Project Goals 


\section{Apply AI techniques and evaluate}

The chosen technique for this project was to apply a specific Artificial Intelligence technique (Fuzzy Logic) to the problem of flight control for a UAV. The control problem was to maintain a desired altitude as shown in Figure 5. Although the main desired output for the aircraft was "Elevator Control", the application was studied further to gain knowledge of the effects of aircraft speed also. This type of application will allow an Intelligent Agent approach to be implemented at a later date.

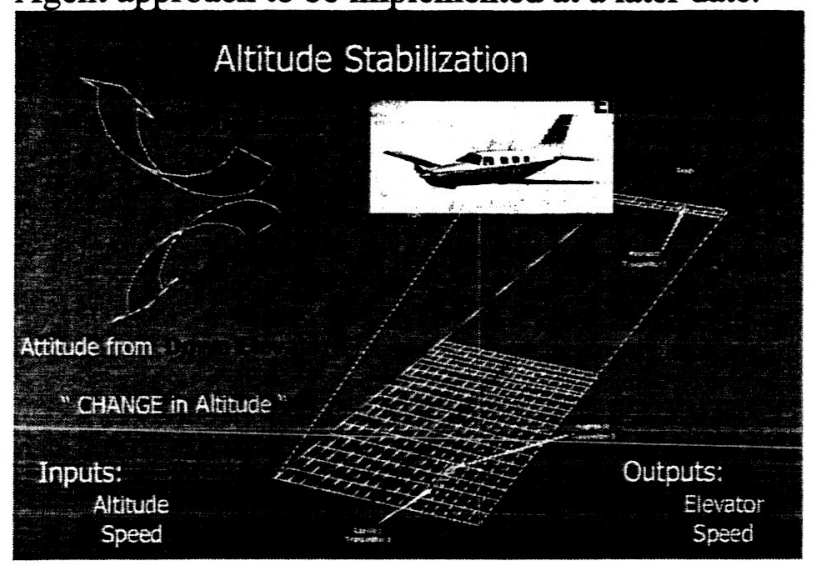

\section{Figure 5. Altitude Control}

The desired project was outlined (Figure 6) but the application chosen for the simulator graphical user interface (GUI) FlightGear proved too complex for the allotted development time. This will be a key application for any research because it provides a visual representation of the UAV control effects. The software is a free distribution and very well supported.

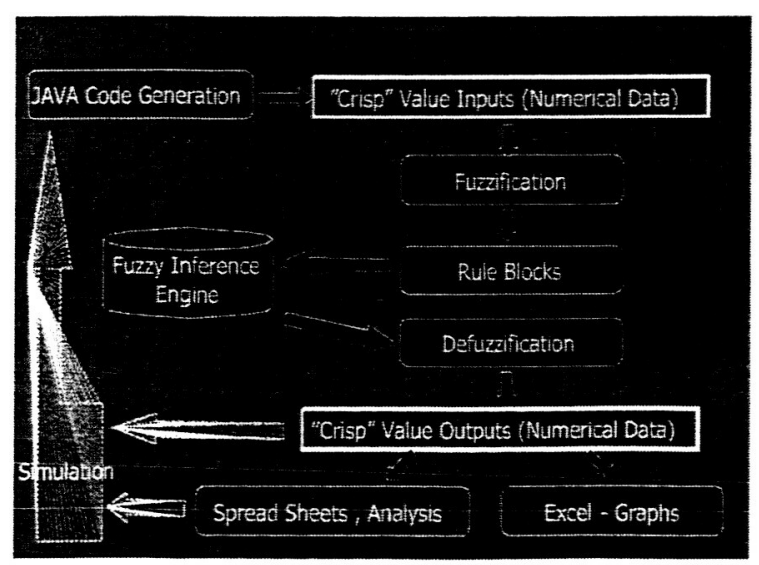

Figure 6. Application of AI Technique

\section{Results}

The work performed is described in the following sections.

\section{Results from tests of the system with appropriate data.}

The results from the first program (modified beautifulcode fuzzy logic demo) were somewhat stable but required tweaking of the Degrees of Membership in the program to provide smoother transitions. The program output was in the form of graphs as depicted in Figure 7. Although the desired output for altitude and elevator was achieved, some experimentation was done with speed. A control input of the desired elevation of $26 \mathrm{~km}$ produced the desired output for speed (normal $=$ nominal throttle for maintaining altitude). The desired elevator output was also achieved (steady $=$ level elevator). The obvious effects of elevator angle will be different depending on the speed the aircraft is moving. This is similar to holding your hand out the window of a moving car. At slow speeds you can turn your flattened hand at different angles and still keep your arm still, but when the speed increases to around $50 \mathrm{MPH}$ your arm becomes unstable and moves wildly about when you increase the angle of attack of your hand.

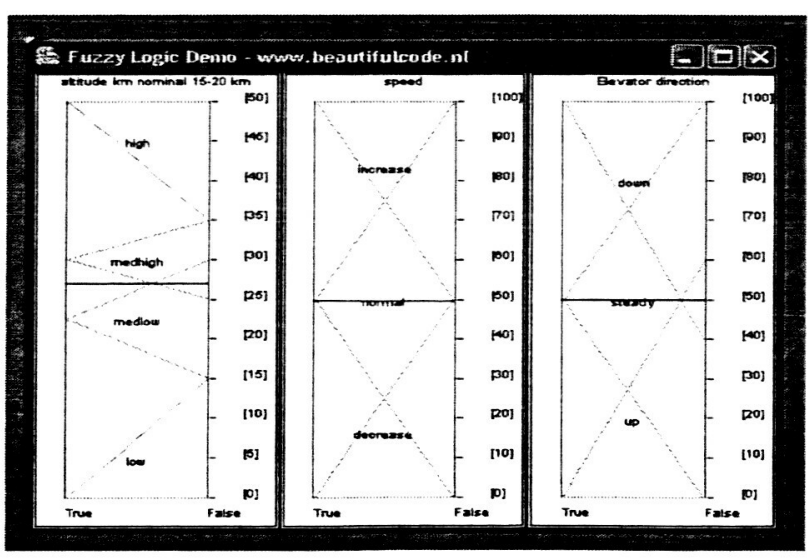

Figure 7. Fuzzy Output Graph

The off-the-shelf program (FuzzyTech demo) was used on a data input file created with a Java program written to give simulated calibrated UAV 
flight data (not real data). FuzzyTech 5.5 was used to design a simple fuzzy controller. The designed fuzzy controller was used to successfully read the data and output a data file. The output file was then read in using another Java program written for it. Microsoft Excel was used to graph the data file. The desired altitude was 5000 feet, and the graph in Figure 8 displays the expected elevator outputs for the generated altitude calibration steps.

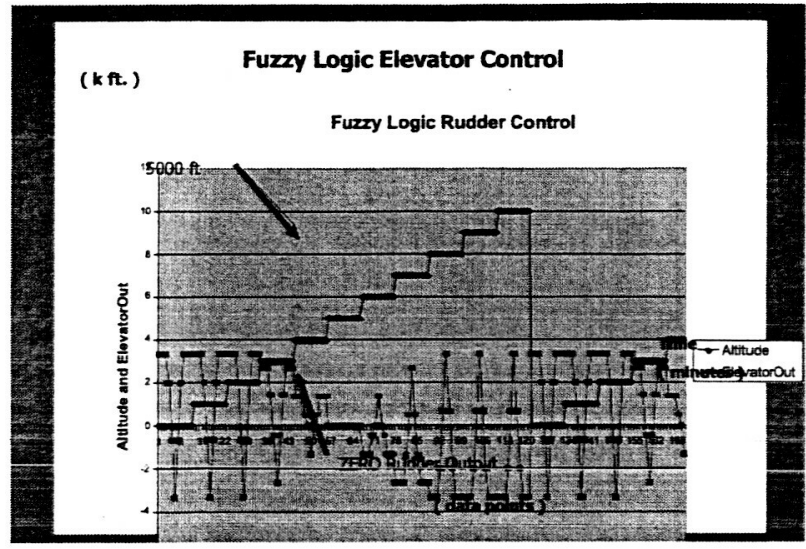

Figure 8. Fuzzy Controller Results

\section{Conclusion and Future Work}

The AI techniques can be greatly expanded with further work in this development. Attempts can be made to expand the use of AI techniques so a comparison can be done against more conventional flight controllers. While this paper described the accomplished work only, much time was spent installing and learning to compile the flight simulator FlightGear. This flight simulator program would provide an excellent interface option for the tools used so far. The completed JAVA code would have to be expanded to use one of the communication protocols that FlightGear provides. The setup and protocol for the communications was the primary blockade during this project assignment.

Once these tools are further developed, the combination of code plus the capability to visually view the performance and analyze the results realtime will provide a strong, practical, and low cost platform for experimentation with AI techniques which are applied to flight control. The summary of some of these conclusions and future work are outlined in Figure 9.

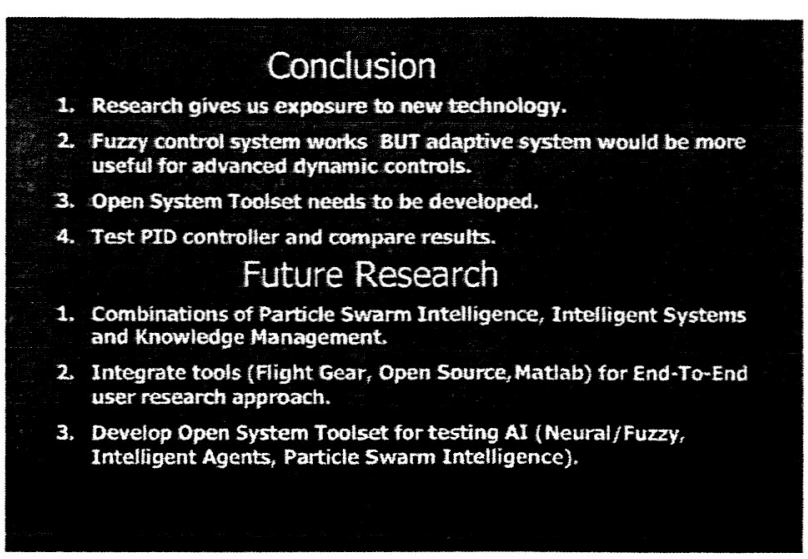

\section{Figure 9. Conclusion and Future Research}

The variety of research being conducted today is vast. Figure 10 illustrates a small part of the variety in UAV systems and parallel bionic types of systems. These types of systems are combining knowledge from our natural environment with software and advancements in technology to produce adaptable systems.

\section{References}

[1] Liu, X., J. Liu, J. Eker, and E.A. Lee, 2002,

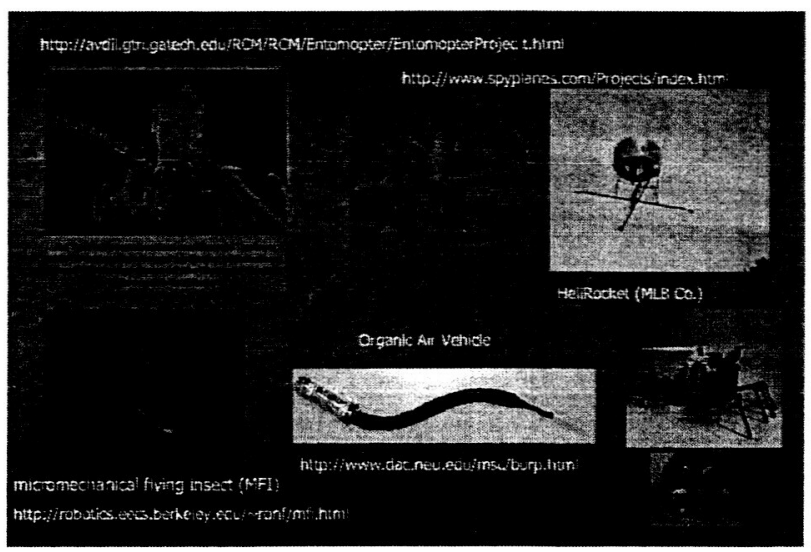

Figure 10. Systems of the Future

"Heterogeneous Modeling and Design of Control Systems," to appear in Software-Enabled Control: Information Technology for Dynamical Systems, T. Samad and G. Balas (eds.), New York City: IEEE Press. 
[2] Cannady, J., 2002, DCIS 760 Artificial Intelligence. (Class Lecture Viewgraphs), Nova Southeastern University, Graduate School of Information Sciences Web site, URL:

http://scis.nova.edu/ cannady [cited January 9, 2003]

[3] Handelman, D., R. Stengel, 1989, "Perspectives on the Use of Rule-Based Control," Artificial Intelligence in Real-Time Control, Pergamon Press, New York, pp. 27-32.

[4] Wyeth G., G. Buskey, J. Roberts, 2000, “Flight Control Using an Artificial Neural Network,"

Proceedings of the Australian Conference on

Robotics and Automation (ACRA 2000), August 30 - September 1, Melbourne.

[5] Page, D., 1998, "MAV Flight Control: Realities and Challenges, " URL:

http://www.hightechcareers.com/doc198e/flightcont rol198e.html [cited January 9, 2003]

[6] Elmore, W., 2000, "Control of Intelligent Unmanned Vehicles in Dynamic Environments,"
URL:

http://www.asinc.com/pdfs/unmannedVehiclesMay 2002.pdf [cited January 9, 2003]

[7] Sehgal, B., R. W. Deters, M.S. Selig, 2002 "Icing Encounter Flight Simulator," AIAA 20020817, Reno, NV, Jan. 2002. 